\title{
Control of Nonlinear Systems with Explicit-MPC-like Controllers
}

\author{
Ivan Pejcic, Milan Korda, and Colin N. Jones
}

\begin{abstract}
This paper describes synthesis of controllers involving Quadratic Programming (QP) optimization problems for control of nonlinear systems. The QP structure allows an implementation of the controller as a piecewise affine function, pre-computed offline, which is a technique extensively studied in the field of explicit model predictive control (EMPC). The nonlinear systems being controlled are assumed to be described by polynomial functions and the synthesis also generates a polynomial Lyapunov function for the closed-loop system involving the obtained controller. The synthesis is based on a sum-of-squares (SOS) stability verification for polynomial discrete-time systems, described in continuous-time in this paper. The presented synthesis method allows a design of EMPC controllers with closed-loop stability guarantees without relying on a terminal cost and/or constraint, and even without using the prediction horizon concept to formulate the control optimization problem. In particular, for a specified QP structure the method directly searches for the stabilizing coefficients in the cost and/or the constraint set. The method involves two phases, where the first searches for stabilizing controllers by minimizing a polynomial slack function introduced to the SOS stability condition and the second phase optimizes some userspecified performance criteria. The two phases are formulated as optimization problems which can be tackled by using a blackbox optimization technique such as Bayesian optimization, which is used in this paper. The synthesis is demonstrated on a numerical example involving a bilinear model of a permanent magnet synchronous machine (PMSM), where in order to demonstrate the modeling flexibility of the proposed synthesis method a QP-based controller for speed regulation of PMSM is synthesized that is robust to parametric uncertainty coming from the temperature-dependent stator resistance of the PMSM.
\end{abstract}

\section{INTRODUCTION}

Model predictive control (MPC) is an optimization-based control technique that has experienced a considerable success due to its ability to handle system constraints [1]. In the case of a (discretized) linear system controlled by an MPC controller whose optimization problem is a parametric Quadratic Program (QP), the version known as explicit MPC (EMPC) stores the offline computed solution of the parametric QP, thus allowing one to avoid running an iterative optimization algorithm during operation.

A key restriction of EMPC is the requirement to utilize a linear dynamic prediction model in the controller formulation, in order to ensure that a (convex) QP is obtained.

Ivan Pejcic and Colin N. Jones are with Laboratoire d'Automatique, École Polytechnique Federale de Lausanne, Switzerland. \{ivan.pejcic, colin.jones\}@epfl.ch.

Milan Korda is with Department of Mechanical Engineering, University of California, Santa Barbara, CA 93106-5070. milan.korda@engineering.ucsb.edu.

The research leading to these results has received funding from the European Research Council under the European Union's Seventh Framework Programme (FP/2007-2013) / ERC Grant Agreement n. 307608: BuildNet.
Moreover, due to the memory limitations EMPC often cannot involve long prediction horizons and is usually applied without the terminal constraint and/or terminal cost which are the elements from MPC theory used to provide guarantees on the stability of the closed-loop system. An alternative approach to establish guaranteed closed-loop stability is an a-posteriori stability verification, which can be performed for a given controller after its design is finished, and which can be done in the case of a discrete-time linear system with QP controller by using the S-procedure [2] or Mixed Integer Linear Programing (MILP) [3]. In the case of a more general class which involves discrete-time systems described by polynomial functions, such a-posteriori stability verification can be done using sum-of-squares (SOS) programming [4].

The synthesis method of this paper develops from the stability verification technique of [4]. It allows the design of EMPC controllers with closed-loop stability guarantees for polynomial systems without relying on a terminal cost and/or constraint, but also even without using the prediction horizon concept to formulate the control optimization problem. In particular, for a specified QP structure the method directly searches for the stabilizing coefficients in the cost and/or the constraint set. This can be regarded as a search for a stabilizing control policy (i.e., a stabilizing mapping from measurements to plant inputs) which is described, in a compressed form, as a parametric QP (a mapping from the QP input parameters to the optimal solution). Although the control synthesis to be presented in this paper is applicable to control structures other than those in EMPC (e.g., a general optimization-based controller whose KKT system is a set of polynomial equalities and inequalities), we present it here with a special focus on the EPMC structure in order to emphasize the possibilities which it creates for that particular popular case.

As will be described in what follows, the synthesis method is based on a reformulation of the SOS stability certification tool [4] and involves two phases. The first phase introduces a slack polynomial function to the stability verification technique and allows a search for stabilizing controllers by a black-box optimization technique, such as Bayesian Optimization which is used in the numerical example of this paper. The second phase takes the stabilizing parameters from the first phase and optimizes for improvement of some user-specified performance criteria, as well by applying Bayesian optimization.

The synthesis of a discrete-time controller for a continuous-time nonlinear system can be done either in discrete-time by using an approximate discrete-time plant model (e.g., obtained by forward Euler discretization) or in 
continuous time by using the continuous-time plant model and a subsequent approximate discrete-time controller implementation with a short sampling time. Although the method of this paper can be developed in both conceptual frameworks, we present it here for the latter case in which the continuous-time nonlinear model is addressed without discretization. For this purpose, we describe in Section II a continuous-time version of the method in [4], which is an additional minor contribution of this paper. The development dealing with discrete-time polynomial systems would involve the discrete-time version from [4]. Section III describes the two phases of the control synthesis method, and Section IV demonstrates it on the bilinear model of a permanent magnet synchronous machine (PMSM).

\section{Continuous-time SOS Stability Verification}

This section describes the continuous-time variation of the discrete-time SOS stability verification from [4]. The obtained stability-certification is a starting point for the development of the control synthesis technique in this paper. Consider a continuous-time polynomial plant model

$$
\begin{aligned}
& \dot{x}=f_{x}(x, u), \\
& y=f_{y}(x),
\end{aligned}
$$

where $x \in \mathbb{R}^{n_{x}}$ is the state vector, $u \in \mathbb{R}^{n_{u}}$ the input vector, $y \in \mathbb{R}^{n_{y}}$ the output vector, $\dot{x} \in \mathbb{R}^{n_{x}}$ the derivative of the state, $f_{x}: \mathbb{R}^{n_{x}+n_{u}} \rightarrow \mathbb{R}^{n_{x}}$ the system function and $f_{y}$ : $\mathbb{R}^{n_{x}} \rightarrow \mathbb{R}^{n_{y}}$ the output mapping. The functions $f_{x}$ and $f_{y}$ are assumed to be vector-valued multivariate polynomials, i.e., each component function of $f_{x}$ and $f_{y}$ is a multivariate polynomial in $(x, u)$ and $x$, respectively. Consider as well an abstract form of the control law defined by the polynomial equalities and inequalities

$$
\begin{aligned}
s & =f_{s}(y ; \eta), \\
\mathbf{K}_{\mathbf{s}} & =\{\theta \mid \exists \lambda \text { s.t. } h(s, \theta, \lambda ; \eta)=0, g(s, \theta, \lambda ; \eta) \geq 0\} \\
u & \in \kappa\left(\mathbf{K}_{s} ; \eta\right),
\end{aligned}
$$

where $s \in \mathbb{R}^{n_{s}}$ is the input to the controller, $\theta \in \mathbb{R}^{n_{\theta}}$ and $\lambda \in \mathbb{R}^{n_{\lambda}}$ internal variables, and the functions $f_{s}: \mathbb{R}^{n_{y}} \rightarrow$ $\mathbb{R}^{n_{s}}, h: \mathbb{R}^{n_{s}+n_{\theta}+n_{\lambda}} \rightarrow \mathbb{R}^{n_{h}}, g: \mathbb{R}^{n_{s}+n_{\theta}+n_{\lambda}} \rightarrow \mathbb{R}^{n_{g}}$, and $\kappa: \mathbb{R}^{n_{\theta}} \rightarrow \mathbb{R}^{n_{u}}$ are vector-valued multivariate polynomials whose coefficients are parametrized in some way by the controller's tuning parameters $\eta \in \mathbb{R}^{n_{\eta}}$.

Remark 1: The discrete-time formulation in reference [4] formulates the problem by including an additional subsystem, which for instance may correspond to an observer. In the formulation given here we omit it in order to keep the expressions shorter.

Remark 2: A QP-based controller can be written in the form (2) by representing its solution with the KKT optimality conditions [5], which are a set of polynomial equalities and inequalities that can be included in $h$ and $g$. Although the focus of this paper is on the QP-based controllers which may lead to an EMPC implementation, we write the control law in the form (2) to emphasise the flexibility of the method which may address a broad variety of other control structures as well.

For selected tuning parameters $\eta$ of the controller, the global closed-loop stability in the state variable $x$ can be certified by a Lyapunov function $V$ satisfying

$$
\begin{array}{r}
-\|x\|_{2}^{2}-\nabla_{x} V(x, \theta, \lambda)^{T} \dot{x} \geq 0, \\
V(x, \theta, \lambda)-\|x\|_{2}^{2} \geq 0 .
\end{array}
$$

Considering the system dynamics (1) and the control law (2), the conditions (3) should be satisfied for all vectors

$$
(x, \theta, \lambda, \dot{x}) \in \mathbf{T}
$$

where the set

$$
\begin{aligned}
\mathbf{T}=\{(x, \theta, \lambda, \dot{x}) \mid & \dot{x}=f_{x}(x, \kappa(\theta ; \eta)), \\
& \hat{h}(x, \theta, \lambda ; \eta)=0, \hat{g}(x, \theta, \lambda ; \eta) \geq 0\}
\end{aligned}
$$

encapsulates the closed-loop system dynamics with the specified control law, and the functions

$$
\begin{aligned}
\hat{h}(x, \theta, \lambda ; \eta) & =h\left(f_{s}\left(f_{y}(x) ; \eta\right), \theta, \lambda ; \eta\right), \\
\hat{g}(x, \theta, \lambda ; \eta) & =g\left(f_{s}\left(f_{y}(x) ; \eta\right), \theta, \lambda ; \eta\right),
\end{aligned}
$$

are introduced to make the notation lighter.

Remark 3: Notice that in the equations (3)-(5) the $\dot{x}$ does not represent the derivative, but actually a variable denoted by $\dot{x}$ whose equality to $f_{x}(x, \kappa(\theta ; \eta))$ is enforced by having the vector of variables $(x, \theta, \lambda, \dot{x})$ in the set $\mathbf{T}$.

Remark 4: The above formulation substitutes the system output $y$, the input to the controller $s$ and the input to the system $u$ by using equations (1b), (2a) and (2c), respectively. This formulation was chosen because it is characterized by a smaller number of equality constraints and allows a simpler exposition of the method. The treatment of the aforementioned relations as additional equality constraints in (5), or elimination of some other variables by their substitution (e.g., elimination of the variable $\dot{x}$ by substituting it with (1a)) are straightforward alternatives.

Remark 5: The conditions (3a) and (3b) involve the terms $\|x\|_{2}^{2}$ whose weighting factors could be optimized as well. In this document, we proceed by keeping them fixed to unity.

The previous formulation involves the nonnegativity conditions (3) imposed over the set (5), which is a problem that can be tackled by using SOS programming. In particular, by denoting

$$
\xi=(x, \theta, \lambda, \dot{x})
$$

and by restricting the Lyapunov function to be a polynomial of a certain (user specified) degree, a sufficient condition for the nonnegativities (3) over the set $\mathbf{T}$ are the following polynomial equalities

$$
\begin{aligned}
&-\|x\|_{2}^{2}-\nabla_{x} V(x, \theta, \lambda)^{T} f_{x}(x, u)=\sigma_{0}(\xi) \\
&+\sigma_{1}(\xi)^{T} \hat{g}(x, \theta, \lambda ; \eta)+p_{1}(\xi)^{T} \hat{h}(x, \theta, \lambda ; \eta) \\
&+p_{2}(\xi)^{T}\left(\dot{x}-f_{x}(x, \kappa(x ; \eta)),\right. \\
& V(x, \theta, \lambda)-\|x\|_{2}^{2}=\bar{\sigma}_{0}(\xi) \\
& \quad+\bar{\sigma}_{1}(\xi)^{T} \hat{g}(x, \theta, \lambda ; \eta)+\bar{p}_{1}(\xi)^{T} \hat{h}(x, \theta, \lambda ; \eta),
\end{aligned}
$$


where the $\sigma_{0}$ and $\bar{\sigma}_{0}$ are SOS polynomials (defined below) with some user specified degrees, $\sigma_{1}, \bar{\sigma}_{1}$ are vectors whose components are SOS polynomials with user specified degrees, and $p_{1}, p_{2}, \bar{p}_{1}$ are vectors of arbitrary polynomials with user specified degrees as well. A polynomial $\sigma(\xi)$ is said to be SOS if there exists a representation

$$
\sigma(\xi)=v(\xi)^{T} P v(\xi),
$$

where $v(\xi)$ is a vector of polynomials and $P \succeq 0$ is a positive semidefinite matrix of appropriate size, resulting in $\sigma(\xi)$ being nonnegative for every $\xi$. The satisfaction of the nonnegativity conditions in (3) over the set (5) follows directly from (8) since for $\xi \in \mathbf{T}$ the SOS polynomials $\sigma$ are nonnegative and the arbitrary polynomials $p$ are equal to zero.

The previous discussion of closed-loop stability verification thus boils down to the feasibility of the SOS problem

$$
\begin{array}{lll}
\text { find } & V, \sigma_{0}, \sigma_{1}, p_{1}, p_{2}, \bar{\sigma}_{0}, \bar{\sigma}_{1}, \bar{p}_{1} \\
\text { s.t. } & (8 \mathrm{a}),(8 \mathrm{~b}), & \\
& \sigma_{0}, \sigma_{1}, \bar{\sigma}_{0}, \bar{\sigma}_{1} & \text { SOS polynomials, } \\
& V, p_{1}, p_{2}, \bar{p}_{1} \quad \text { arbitrary polynomials, }
\end{array}
$$

where the decision variables are the coefficients of the polynomials $\left(V, \sigma_{0}, \sigma_{1}, p_{1}, p_{2}, \bar{\sigma}_{0}, \bar{\sigma}_{1}, \bar{p}_{1}\right)$. This problem converts to a semidefinite programming (SDP) convex optimization problem, and can thus be solved efficiently. The conversion can be done automatically by using freely available software like Yalmip [6]. For more information about the conversion of (10) to SDP, the reader is referred to, e.g., [7], [8].

Remark 6: Although the method extends to the case with reference tracking, we formulate it here for the case of regulation of the state $x$ to the origin of the state-space in order to avoid cumbersome expressions and better emphasize the fundamental concepts of the method. The application of the method to the case involving reference tracking in delta formulation is demonstrated in the computational example section.

While the SOS program (10) ensures global stability in the state variable $x$, the method can also be modified to address local stability over a set

$$
\mathbf{X}=\left\{x \mid \psi_{i} \geq 0, i=1, \ldots, n_{\psi}\right\} .
$$

This is done by including the inequalities of the set $\mathbf{X}$ in the set $\mathbf{T}$, and by subsequently assigning them SOS polynomial multipliers (since they are inequality constraints) in (8) as well. The satisfaction of such a modified condition (8) however does not guarantee invariance of the closedloop system over the whole set $\mathbf{X}$, but only over the largest sublevel set of the Lyapunov function which is contained in $\mathbf{X}$, likewise in the case of discrete-time version in [4].

\section{Controller Synthesis}

An attempt to involve the controller tuning parameters $\eta$ as decision variables in the SOS programming stability verification (10) makes the problem loose the SDP structure, since after the conversion the constraint (8) would now be equivalent to a Bilinear Matrix Inequality (BMI) instead of to a Linear Matrix Inequality (LMI). The optimization problem would thus end up in a form which is in general challenging to solve.

The synthesis optimization problem addressed in what follows can be formulated as

$$
\begin{array}{ll}
\min . & \mathrm{P}(\eta)+\delta_{s t}(\eta) \\
\text { s.t. } & \eta \in \mathbf{D},
\end{array}
$$

where the $\delta_{s t}(\eta)$ is a function indicating the existence of the SOS stability certificate from (10):

$$
\delta_{s t}(\eta)= \begin{cases}0, & \text { for } \eta \text { with a stability certificate, } \\ +\infty, & \text { otherwise, }\end{cases}
$$

the $\mathrm{P}(\eta)$ is a user-specified performance criteria for the closed-loop system with control parameters $\eta$, and the set $\mathbf{D}$ models some basic requirements on the tuning parameters $\eta$ (e.g., a requirement that the Hessian in the cost function of a QP-based controller is symmetric positive definite)

The solving of optimization problem (12) in what follows consists of two phases. The first phase searches for feasible (i.e., stabilizing) control parameters $\eta$ in (12). This is achieved by introducing a slack polynomial function into the stability certification constraint (8) (described in Section III-A) and then by minimizing its presence by using Bayesian optimization to obtain parameters $\eta$ feasible in (12) (described in Section III-B). The second phase takes the generated stabilizing tuning parameters of (12) as initial conditions which are providing an indication about the location of a stabilizing region, and then starting from that data further explores for improvement of the performance criteria $\mathrm{P}(\eta)$ by means of Bayesian optimization and its data exploitation property (described in Section III-C).

\section{A. Reformulation with slack polynomial function}

To allow controller synthesis, we introduce an additional SOS polynomial in the condition (8a), denoted $\sigma_{\mathrm{sl}}(\xi)$ and of the same degree as the $\sigma_{0}(\xi)$, in order to play the role of a slack, which results in the constraint (8a) taking the form:

$$
\begin{aligned}
-\|x\|_{2}^{2} & -\nabla_{x} V(x, \theta, \lambda)^{T} f_{x}(x, u)=\sigma_{0}(\xi)-\sigma_{\mathrm{sl}}(\xi) \\
& +\sigma_{1}(\xi)^{T} \hat{g}(x, \theta, \lambda ; \eta)+p_{1}(\xi)^{T} \hat{h}(x, \theta, \lambda ; \eta) \\
& +p_{2}(\xi)^{T}\left(\dot{x}-f_{x}(x, \kappa(x ; \eta)),\right.
\end{aligned}
$$

while the constraint (8b) will be retained without modification. Since any arbitrary polynomial can be written as a difference of two SOS polynomials [9], the $\sigma_{0}(\xi)-\sigma_{\mathrm{sl}}(\xi)$ term can express any arbitrary polynomial up to the degree of $\sigma_{0}(\xi)$ and $\sigma_{\mathrm{sl}}(\xi)$. Thus, the constraint consisting of (14) and (8b) has a feasible solution for any fixed value of the parameter $\eta$, provided that the degree of $\sigma_{0}(\xi)$ and $\sigma_{\mathrm{sl}}(\xi)$ is no smaller than the degree of the other polynomials in (14).

Since the goal will be to minimize the presence of the slack as much as possible to make it become identically equal to zero, consider a cost function which is an integral of the SOS slack polynomial $\sigma_{\mathrm{sl}}(\xi)$. In particular, for $\sigma_{\mathrm{sl}}(\xi)=$ $\sum_{i=1}^{n_{\beta}} v_{i} \beta_{i}(\xi)$ where $v_{i}$ are the polynomial's coefficients and 
$\left(\beta_{i}\right)_{i=1}^{n_{\beta}}$ the corresponding monomials, the integral over some simple set $\mathbf{Y}$ (like for instance a unit box which will be used in the numerical example) is:

$$
\int_{\mathbf{Y}} \sigma_{\mathrm{sl}}(\xi) d \xi=\sum_{i=1}^{n_{\beta}} v_{i} \int_{\mathbf{Y}} \beta_{i}(\xi) d \xi
$$

and is a linear function in the coefficients $v_{i}$ weighted by the integrals of the $\beta_{i}$ monomials over the set $\mathbf{Y}$ (these integrals are constant values).

Since this cost is a linear function in the coefficients of the SOS polynomial $\sigma_{\mathrm{sl}}(\xi)$, together with the constraint set containing (14) and (8b) it represents the SOS problem:

$$
\begin{array}{cll}
\mathrm{I}_{\sigma}(\eta)=\min . & \int_{\mathbf{Y}} \sigma_{\mathrm{sl}}(\xi) d \xi & \\
\text { s.t. } & (14),(8 \mathrm{~b}), & \\
& \sigma_{\mathrm{sl}}, \sigma_{0}, \sigma_{1}, \sigma_{2}, \bar{\sigma}_{0}, \bar{\sigma}_{1} & \text { SOS polynomials, } \\
& V, p_{1}, p_{2}, p_{3}, \bar{p}_{1} & \text { arbitrary polynomials, }
\end{array}
$$

which corresponds to an SDP for any fixed $\eta$, and $\mathrm{I}_{\sigma}(\eta)$ is the optimal value of the problem. As $\sigma_{\mathrm{sl}}(\xi)$ is an SOS polynomial and thus globally nonnegative, the integral $\mathrm{I}_{\sigma}(\eta)$ is zero only when the polynomial $\sigma_{\mathrm{sl}}(\xi)$ is identically equal to zero. This $\mathrm{I}_{\sigma}(\eta)=0$ corresponds to the case involving $\eta$ for which the stability certificate from (10) exists. Otherwise, the slack function $\sigma_{\mathrm{sl}}(\xi)$ (and its corresponding integral $\mathrm{I}_{\sigma}(\eta)$ ) are non-zero and minimization of $\mathrm{I}_{\sigma}(\eta)$ as a function of $\eta$ would lead to stabilizing controller parameters (i.e., those satisfying $\mathrm{I}_{\sigma}(\eta)=0$ ), as discussed next.

\section{B. Search for stabilizing control parameters}

Let $\mathbf{D}$ be the set of tuning parameters $\eta$ satisfying some basic design requirements, as defined in (12). The set containing the tuning parameters $\eta$ with SOS stability certificate is $\left\{\eta \mid \mathrm{I}_{\sigma}(\eta)=0, \eta \in \mathbf{D}\right\}$. In the case when it is nonempty, it corresponds to the set of optimal solutions of the optimization problem

$$
\begin{array}{ll}
\min . & \mathrm{I}_{\sigma}(\eta) \\
\text { s.t. } & \eta \in \mathbf{D} .
\end{array}
$$

In case of $\left\{\eta \mid \mathrm{I}_{\sigma}(\eta)=0, \eta \in \mathbf{D}\right\}$ being empty, the optimal value of (17) would be larger than zero and it is not possible to find tuning parameters $\eta$ with stability certificate (10).

The minimization (17) that leads to stabilizing tuning parameters $\left\{\eta \mid \mathrm{I}_{\sigma}(\eta)=0, \eta \in \mathbf{D}\right\}$ can be performed by using a black-box global optimization method. In this paper, we use Bayesian optimization which is a derivative-free method for finding a constrained global optimal solution of a black-box cost function. The constraint set can be specified either explicitly (like the set D in (17)) or as an error in the evaluation of the cost (i.e., the value $+\infty$ returned by cost function), and the values of the cost function are allowed to be either deterministic or stochastic (see [10] for information pertaining to the practical aspects of the method). The algorithm is conceived in such a way that at each iteration of the Bayesian optimization method, the currently available cost evaluation pairs $\left\{\eta_{i}, \mathrm{I}_{\sigma}\left(\eta_{\mathrm{i}}\right)\right\}$ are used to build a statistical model of the cost function based on Gaussian Processes. This model is then employed to construct an acquisition function $a(\eta)$, which is such that its minimizer represents the next sampling point $\eta$ that balances between exploitation of the currently known cost values and exploration of the less known regions of the cost function $\mathrm{I}_{\sigma}(\eta)$. The feature that the next sampling point $\eta$ is determined by minimizing the acquisition function $a(\eta)$ instead of operating with the actual cost function $\mathrm{I}_{\sigma}(\eta)$ makes the method particularly suitable for problems where the evaluation of the cost function $\mathrm{I}_{\sigma}(\eta)$ is time consuming or in some other sense expensive.

\section{Optimization of performance}

The optimization problem (12) can be addressed by Bayesian Optimization, which would treat the $+\infty$ values from $\delta_{s t}(\eta)$ as the error in the evaluation of the cost. A problem however is that in the initial phase before any tuning parameters with SOS stability certificate are found (i.e., any parameter $\eta$ with $\delta_{s t}(\eta)=0$ ), the Bayesian optimization would have only values $+\infty$ available, which are not very informative for choosing where to sample $\eta$ next in order to reach a region with stabilizing parameters. For this reason, the solving of (12) by Bayesian optimization should be preceded by a search for stabilizing tuning parameters with (17), so that after a certain number (e.g., twenty) of stabilizing tuning parameters $\eta$ is found by (17) one can use them as initial points to start Bayesian optimization on (12). These intial points (which would be different among themselves due to the exploration property) would provide some information to the Bayesian optimization solving (12) about the location of the stabilizing region in the space of tuning parameters, and by the exploitation property of Bayesian optimization it would be a region of focus for further investigation while minimizing the performance criteria $\mathrm{P}(\eta)$.

There is a great amount of flexibility in the choice of the cost term $\mathrm{P}(\eta)$ in (12), as it is allowed to be any performance criteria which can be evaluated for a fixed vector of tuning parameters $\eta$. A possible broadly applicable choice is an approximate evaluation of the integral of the infinite horizon trajectory cost over some set $\mathbf{W}$ :

$\mathrm{P}(\eta)=\int_{\mathbf{W}} C_{\infty, \eta}(x) d x, C_{\infty, \eta}(x)=\int_{0}^{\infty} l(x(t), u(t)) d t$,

where $C_{\infty, \eta}(x)$ is the infinite horizon trajectory cost obtained with controller $\eta$ when starting from the state $x$, and $l(x, u)$ is some stage cost. Equation (18) can be evaluated approximately by using Monte Carlo (MC) approximation for the integral and finite horizon approximations for the trajectory costs obtained from a discretized version of the continuoustime system:

$$
\mathrm{P}(\eta)=\sum_{j=1}^{N_{m c}} \tilde{C}_{N_{s t}, \eta}\left(x_{j}\right), \quad \tilde{C}_{N_{s t}, \eta}(x)=T_{s} \sum_{k=0}^{N_{s t}} l\left(x_{k}, u_{k}\right),
$$


where $T_{s}$ is the sampling time of the discrete-time simulation of the continuous-time system (obtained by applying the forward Euler method for example), $x_{k}$ and $u_{k}$ are the state and input values at the $k$-th simulation step, the $\tilde{C}_{N_{s t}, \eta}(x)$ is the finite horizon trajectory cost involving $N_{s t}$ simulation steps from the initial state $x$, and the $N_{m c}$ is the number of samples from the set $\mathbf{W}$ in the $\mathrm{MC}$ approximation of the integral. As the evaluation of $\mathrm{P}(\eta)$ involves $\mathrm{MC}$ approximation, the Bayesian Optimization should consider the cost function values as stochastic.

\section{Computational Example}

This section demonstrates the control synthesis method by an example involving a QP-based controller for control of a bilinear system with parameter uncertainty. The SOS programming problems are implemented by using Yalmip [6] as a modelling tool together with MOSEK as SDP solver, and the Bayesian optimization is applied by using Matlab's Statistics and Machine Learning Toolbox [10].

A. QP-based controller for a bilinear system with parametric uncertainty

This section synthesises a QP-based controller for speed control of a permanent magnet synchronous machine (PMSM). The small size of the QP control structure used in this section allows its implementation for the control of a bilinear PMSM model in EMPC fashion. In comparison to the MPC scheme for PMSM developed in [11], the controller synthesis of this section directly deals with the bilinear model of the system, thus circumventing the need for using a linear discrete-time prediction model like in [11] which is valid only at nominal (or some other fixed and in advance chosen) rotational speed. Furthermore, the controller presented here is synthesized robust to the stator resistance variations caused by temperature changes, and also without the outer speedcontrol loop based on an additional PI controller, which are features both mentioned in [11] as desirable to be addressed in future research.

The continuous-time model of the two-pole PMSM in the $d q$ reference frame fixed to the rotor (see, e.g., [12]) has the form

$$
\begin{aligned}
\frac{d I_{d}(t)}{d t} & =-\frac{\tilde{R}_{s}}{L_{s}} I_{d}(t)+\Omega_{r}(t) I_{q}(t)+\frac{1}{L_{s}} U_{d}(t), \\
\frac{d I_{q}(t)}{d t} & =-\frac{\tilde{R}_{s}}{L_{s}} I_{q}(t)-\left(I_{d}(t)+\frac{\Phi_{0}}{L_{s}}\right) \Omega_{r}(t)+\frac{1}{L_{s}} U_{q}(t), \\
\frac{d \Omega_{r}(t)}{d t} & =\frac{K_{t}}{J} I_{q}(t)-\Gamma_{L}(t),
\end{aligned}
$$

where $I_{d}(t)$ and $I_{q}(t)$ are the $d$ and $q$ component of the stator current vector $I(t)=\left[I_{d}(t), I_{q}(t)\right]^{T}, \Omega_{r}(t)$ is the rotational speed of the rotor, $U_{d}(t)$ and $U_{q}(t)$ are the $d$ and $q$ component of the input voltage vector $U(t)=\left[U_{d}(t), U_{q}(t)\right]^{T}$, $\Gamma_{L}(t)$ is the load torque, the parameter $\tilde{R}_{s}$ is stator resistance, $L_{s}$ stator inductance, $\Phi_{0}$ the flux from the rotor's permanent magnet, $K_{t}$ torque coefficient and $J$ the rotational inertia of the rotor. The value of stator resistance $\tilde{R}_{s}$ is characterised by slow variations caused by the changes in temperature,
TABLE I

PMSM PARAMETERS AND NOMINAL VALUES, BASE VALUES FOR THE PER-UNIT SYSTEM, AND THE PER-UNIT PMSM PARAMETERS.

\begin{tabular}{|l|lr|}
\hline Name & Notation & Value \\
\hline \hline Stator resistance & $R_{s}$ & $4.3 \Omega$ \\
Stator inductance & $L_{s}$ & $3.56 \mathrm{mH}$ \\
Flux from rotor & $\Phi_{0}$ & $0.0245 \mathrm{~Wb}$ \\
Torque coefficient & $K_{t}$ & $36.8 \mathrm{mNm} / \mathrm{A}$ \\
Rotational inertia & $J$ & $11 \cdot 10^{-7} \mathrm{Nm}$ \\
Nominal (phase) voltage & $U_{\mathrm{nom}}$ & $36 / \sqrt{3} \mathrm{~V}$ \\
Nominal current & $I_{\mathrm{nom}}$ & $0.8 \mathrm{~A}$ \\
Nominal torque & $T_{\mathrm{nom}}$ & $30 \mathrm{mNm}$ \\
Base value of voltage & $U_{b}=U_{\mathrm{nom}}$ & $36 / \sqrt{3} \mathrm{~V}$ \\
Base value of current & $I_{b}=I_{\mathrm{nom}}$ & $0.8 \mathrm{~A}$ \\
Base value of impedance & $Z_{b}=U_{\mathrm{nom}} / I_{\mathrm{nom}}$ & $25.98 \Omega$ \\
Base value of speed & $\omega_{b}$ & $5000 \cdot 2 \pi \mathrm{rad} / \mathrm{s}$ \\
Base value of rotor's flux & $\Phi_{b}=U_{\mathrm{nom}} / \omega_{b}$ & $0.0397 \mathrm{~Wb}$ \\
Per-unit stator resistance & $r_{s}=R_{s} / Z_{b}$ & 0.4138 \\
Per-unit stator inductance & $l_{s}=\omega_{b} L_{s} / Z_{b}$ & 0.0717 \\
Per-unit flux from rotor & $\phi_{0}=\Phi_{0} / \Phi_{b}$ & 0.6172 \\
Per-unit torque constant & $\mathcal{K}_{t}=\left(K_{t} I_{b}\right) /\left(J \omega_{b}^{2}\right)$ & 51.11 \\
\hline
\end{tabular}

leading to the values of $\tilde{R}_{s}$ which during operation can be several times larger than the $R_{s}$ contained in Table I where the parameters of PMSM from [11] are given. The system has an input constraint concerning the magnitude of the input voltage vector:

$$
\|U(t)\|_{2} \leq U_{n o m}
$$

while due to the thermal inertia of the machine, the stator current vector $I(t)$ is allowed to make temporary violations of the constraint $\|I(t)\|_{2} \leq I_{\text {nom }}$ during transients, but still not of an excessively large magnitude which could cause damage on the machine or voltage source (e.g., it should be ensured that $\left.\|I(t)\|_{2} \leq 5 I_{\text {nom }}\right)$.

By using the base values in Table I, the following normalized model is obtained:

$$
\begin{aligned}
& \frac{d i_{d}(\tau)}{d \tau}=-\frac{\tilde{r}_{s}}{l_{s}} i_{d}(\tau)+\omega_{r}(\tau) i_{q}(\tau)+\frac{1}{l_{s}} u_{d}(\tau) \\
& \frac{d i_{q}(\tau)}{d \tau}=-\frac{\tilde{r}_{s}}{l_{s}} i_{q}(\tau)-\left(i_{d}(\tau)+\frac{\phi_{0}}{l_{s}}\right) \omega_{r}(\tau)+\frac{1}{l_{s}} u_{q}(\tau)
\end{aligned}
$$

$$
\frac{d \omega_{r}(\tau)}{d \tau}=\mathcal{K}_{t} i_{q}(\tau)-\frac{T_{b}}{J \omega_{b}^{2}} \gamma_{L}(\tau),
$$

where $\tau=\omega_{b} t$ is per-unit time, $i_{d}(\tau)=I_{d}(t) / I_{b}, i_{q}(\tau)=$ $I_{q}(t) / I_{b}$ are per-unit stator current components, $\omega_{r}(\tau)=$ $\Omega_{r}(t) / \omega_{b}$ per-unit rotational speed, $u_{d}(\tau)=U_{d}(t) / U_{b}$, $U_{q}(\tau)=U_{q}(t) / U_{b}$ per-unit input voltage components, $\gamma_{L}(\tau)=\Gamma_{L}(t) / T_{b}$ per-unit load torque, and the per-unit parameters appearing in the model are as defined in Table I. The input constraint for the per-unit model takes the form $\|u(\tau)\|_{2} \leq 1$, and the constraint on the current which can be temporarily violated during transients $\|i(\tau)\|_{2} \leq 1$. The corresponding state vector is $x(\tau)=\left[i_{d}(\tau), i_{q}(\tau), \omega_{r}(\tau)\right]^{T}$ and the input vector $u(\tau)=\left[u_{d}(\tau), u_{q}(\tau)\right]^{T}$. It can be seen that the plant model is bilinear as it involves products of the state variables. 
For the purpose of tracking a constant speed reference $r$, the steady-state target operating point $x_{s}=\left[i_{d s}, i_{q s}, \omega_{r s}\right]^{T}$, $u_{s}=\left[u_{d s}, u_{q s}\right]^{T}$ at which the rotational speed is equal to $r$ is to be computed. In order to keep the synthesis example simpler and avoid additional complications, we consider the case involving a zero load torque (i.e., $\gamma_{L}=0$ ) which results in the steady-state target operating point of the form

$$
x_{s}=\left[\begin{array}{l}
0 \\
0 \\
r
\end{array}\right], \quad u_{s}=\left[\begin{array}{c}
0 \\
\phi_{0} r
\end{array}\right] .
$$

Consideration of a non-zero load torque, such as for example a $\gamma_{L}$ which is a polynomial function of the rotational speed or a constant $\gamma_{L}$ whose value is provided to the controller by an estimator, is also possible and reflects itself on the expression for the steady-state target (23).

The QP-based controller will be synthesised so that the QP takes as its inputs the target $u_{s}$ and the deviation from the steady-state target $\Delta x=x-x_{s}$, and provides as its output (its optimal solution) the deviation $\Delta u$ from the steady-state target $u_{s}$ (i.e., the input signal to the PMSM is $u=u_{s}+\Delta u$ ). The form of the QP is selected to be

$$
\begin{array}{ll}
\min . & \frac{1}{2} z^{T} H z+\Delta x^{T} F z \\
\text { s.t. } & G z \leq d-G u_{s},
\end{array}
$$

where $z \in \mathbb{R}^{2}$ is the decision vector, $H \in \mathbb{R}^{2 \times 2}$ is a symmetric positive definite matrix, $F \in \mathbb{R}^{3 \times 2}$, and since the optimal value of (24) corresponds to the input deviation $\Delta u$, the constraint matrices $G \in \mathbb{R}^{6 \times 2}$ and $g \in \mathbb{R}^{6}$ are chosen such that they approximate the input constraint $\| u_{s}+$ $\Delta u \|_{2} \leq 1$ by an inner polytopic approximation consisting of $n_{h}=6$ halfspaces $g_{i}^{T} z \leq d_{i}, \forall i \in\left\{1, \ldots, n_{h}\right\}$ where $g_{i}=\left[\cos \left(\pi i / n_{h}\right), \sin \left(\pi i / n_{h}\right)\right]^{T}, d_{i}=\cos \left(\pi / n_{h}\right)$, as can be seen on Fig. 2. The controller's tuning parameters (the vector $\eta$ ) are the elements of the $H$ and $F$ matrix, thus resulting in $\eta \in \mathbb{R}^{9}$ (due to the symmetry of $H$ ).

To represent the solution of the QP (24) as a system of polynomial equalities and inequalities, consider its corresponding KKT system [5]:

$$
\begin{aligned}
H z+F^{T} \Delta x+G^{T} \lambda & =0, \\
\lambda^{T}\left(G z-d+G u_{s}\right) & =0, \\
d-G z-G u_{s} & \geq 0, \\
\lambda & \geq 0,
\end{aligned}
$$

where $\lambda \in \mathbb{R}^{6}$ is a dual variable, (25a) represents the stationarity condition, (25b) is complementarity slackness, (25c) is primal feasibility and (25d) dual feasibility. The existence of $\lambda$ so that (25) is satisfied by some $\theta$ is a necessary and sufficient condition for that $\theta$ to be optimal in (24), provided some constraint qualification conditions are satisfied, which is the case for (24) with the specified $G$ and $d$ (for a detailed treatment of optimality conditions in convex optimization, see e.g. [13]).

For the controller synthesis, the vector of variables is selected to be

$$
\xi=\left(r, \Delta x, \lambda, \Delta \dot{x}, \tilde{r}_{s}\right)
$$

and all other variables are expressed as a function of $\xi$. In particular, at all places at which they appear, the $x_{s}$ and $u_{s}$ are expressed as in (23), the $x$ and $u$ as $x=x_{s}+\Delta x$ and $u=u_{s}+\Delta u$, respectively, the $\Delta u$ as $\Delta u=z$, and the $z$ as $z=-H^{-1}\left(F^{T} \Delta x+G^{T} \lambda\right)$ which is obtained from (25a).

For the purpose of reference tracking, the Lyapunov conditions (3) are formulated in delta space and have the form

$$
\begin{array}{r}
\Delta x^{T} \Delta x-\nabla_{\Delta x} V\left(\Delta x, r, \tilde{r}_{s}\right)^{T} \Delta \dot{x} \geq 0, \\
V\left(\Delta x, r, \tilde{r}_{s}\right)-\Delta x^{T} \Delta x \geq 0 .
\end{array}
$$

It can be seen that the Lyapunov function $V\left(\Delta x, r, \tilde{r}_{s}\right)$ depends on the speed reference $r$ (specified below to be in the range $[-1,1]$ ) and on the stator resistance $\tilde{r}_{s}$ (specified below to be in the range $\left[r_{s}, 5 r_{s}\right]$, where $r_{s}$ is as stated in Table I). For control law (2), by selecting the input parameter $s=\left[\Delta x, u_{s}\right]^{T}$ and the internal variable $\theta=z$, the $h(s, z, \lambda)$ and $g(s, z, \lambda)$ polynomials take the form

$$
\begin{aligned}
& h(s, z, \lambda)=\left[\lambda^{T}\left(G u_{s}+G z-d\right)\right], \\
& g(s, z, \lambda)=\left[\begin{array}{c}
d-G\left(u_{s}+z\right) \\
\lambda
\end{array}\right] .
\end{aligned}
$$

The local stability certification set (11) is used to incorporate the bound on the reference $|r| \leq r_{\max }$ with $r_{\max }=1$, the bound on the state vector $-x_{\max } \leq x_{s}+\Delta x \leq x_{\max }$ with $x_{\max }=[5,5,2]^{T}$, and the bound on the stator resistance $r_{s, \min } \leq \tilde{r}_{s} \leq r_{s, \max }$ with $r_{s, \min }=r_{s}$ and $r_{s, \max }=5 r_{s}$ where $r_{s}$ is the parameter from Table I. The set (11) is thus defined by

$$
\psi=\left[\begin{array}{c}
r+r_{\max } \\
-r+r_{\max } \\
x_{s}+\Delta x+x_{\max } \\
-x_{s}-\Delta x+x_{\max } \\
\tilde{r}_{s}-r_{s, \min } \\
-\tilde{r}_{s}+r_{s, \min }
\end{array}\right]
$$

The control synthesis is run with the Lyapunov function $V\left(\Delta x, r, \tilde{r}_{s}\right)$ of order 4 , SOS $\sigma$ and arbitrary $p$ polynomial multipliers of order 2 , and the SOS slack polynomial $\sigma_{\mathrm{sl}}$ of order 4. The search ranges for tuning parameters in $\eta$ are chosen to be $[-1,1]$ for each component, which is together with the positive-definiteness constraint for the matrix $H$ embedded into the set D. After 120 Bayesian Optimization iterations applied to (17) for the search of stabilizing solutions (involving a unit box as the set $\mathbf{Y}$ in (15)), 17 stabilizing tuning parameters $\eta$ (i.e., vectors in the set $\left\{\eta \mid \mathrm{I}_{\sigma}(\eta)=0, \eta \in \mathbf{D}\right\}$ ) were obtained, with an average time per Bayesian optimization iteration of about 9.5 minutes. These stabilizing parameters were then used as initial points in a total of 240 Bayesian Optimization iterations applied to the performance optimization problem (12), with the average time per iteration slightly larger than 3 minutes. The performance criteria used was the MC approximation of the integral of trajectory costs (19). For it, the trajectories were simulated using forward Euler discretization with $T_{s}=100 \mu s$ (equivalent in per-unit to 

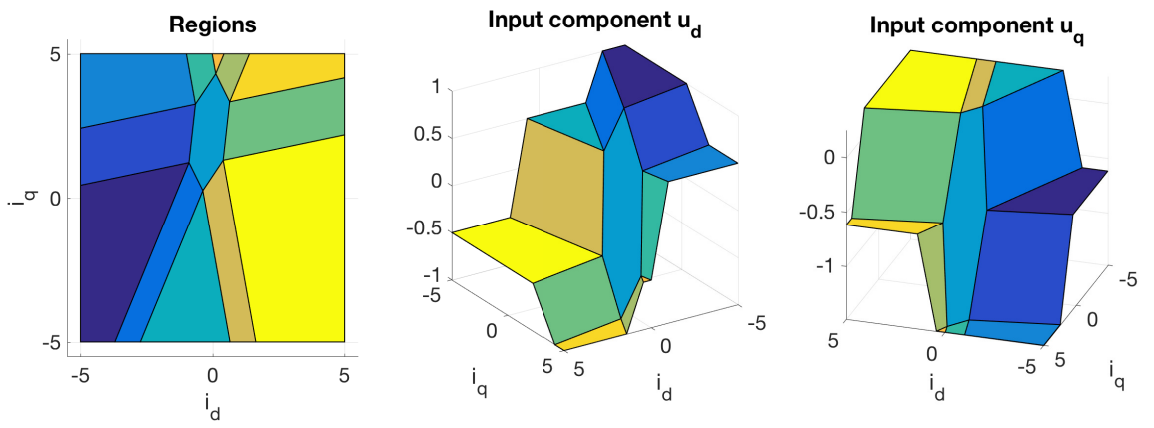

Fig. 1. The EMPC regions and the input components $u=\left[u_{d}, u_{q}\right]^{T}$ obtained with $\omega$ and $r$ fixed to $\omega=0, r=1$. As can be seen, the number of regions of the EMPC is 13 .
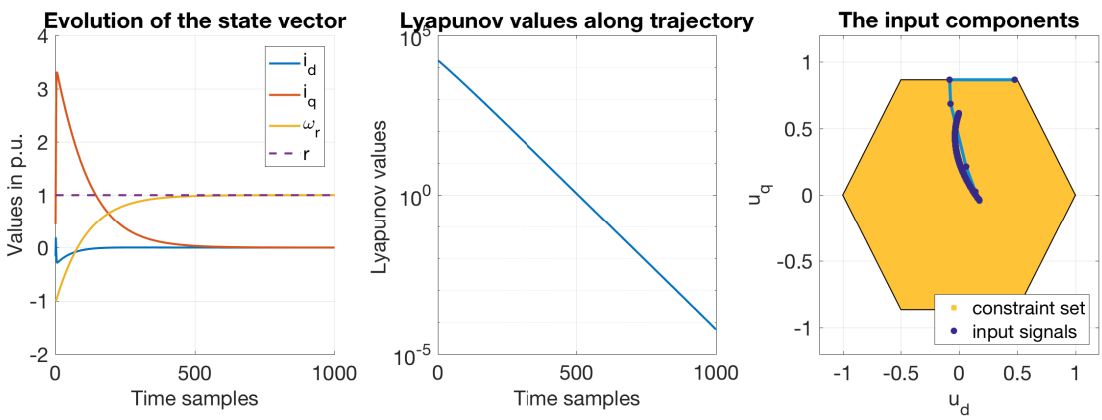

Fig. 2. Evolution of the state vector $x$ starting from the initial $x_{0}=[-0.166,0.441,-0.998]^{T}$ for the reference $r=1$ and resistance $r_{s}=1$, the corresponding Lyapunov values along the trajectory, and the input vectors $u=\left[u_{d}, u_{q}\right]^{T}$ along the trajectory (the target input is $u_{s}=[0,0.617]^{T}$ ).

$\left.\tau_{s}=\omega_{b} T_{s}\right)$ with the value of $\tilde{r}_{s}$ fixed to $\tilde{r}_{s}=2.5 r_{s}$. The $N_{\text {st }}$ was selected to $N_{\mathrm{st}}=500$, and $N_{\mathrm{MC}}$ to $N_{\mathrm{MC}}=90$ which was spread equally for the reference values $r=0$, $r=0.8$ and $r=-0.8$. The stage cost was selected to be

$$
l\left(x_{k}, u_{k}\right)=\Delta x_{k}^{T} Q_{s c} \Delta x_{k}+q_{s c}\left\|u_{k-1}-u_{k}\right\|_{2}^{2},
$$

where $Q_{s c} \in \mathbb{R}^{3 \times 3}$ is diagonal with $2,0.5$ and 1 on its diagonal, and $q_{s c}=1$. The set $\mathbf{W}$ used for initial states in (19) was selected identical to the locality constraint (29).

A slice of the control law with the obtained $H$ and $F$ matrix is represented on Fig. 1 with $\omega$ and $r$ fixed to $\omega=0$, $r=1$. The controller was tested in simulation for various values of $\tilde{r}_{s} \in[1,5]$ by applying it with the sampling time $T_{s}=100 \mu s$ and starting it from many random initial points. Despite the discrete-time application of the controller, the sampling time $T_{s}=100 \mu \mathrm{s}$ was sufficiently small for a good approximation and during testing no violations of the Lyapunov decrease were observed. Fig. 2 shows the state trajectory, the Lyapunov function values and the input signals obtained with speed reference $r=1$ and a randomly generated initial state.

\section{REFERENCES}

[1] J. B. Rawlings and D. Q. Mayne, Model predictive control: Theory and design. Nob Hill Pub., 2009.

[2] J. A. Primbs, "Brief the analysis of optimization based controllers," Automatica, vol. 37, no. 6, pp. 933-938, Jun. 2001.

[3] D. Simon and J. Löfberg, "Stability analysis of model predictive controllers using mixed integer linear programming," arxiv.org, 2016. [Online]. Available: https://arxiv.org/abs/1604.00863
[4] M. Korda and C. N. Jones, "Stability and performance verification of optimization-based controllers," arxiv.org, 2015. [Online]. Available: https://arxiv.org/abs/1501.03919

[5] S. Boyd and L. Vandenberghe, Convex Optimization. New York, NY, USA: Cambridge University Press, 2004.

[6] J. Löfberg, "Yalmip : A toolbox for modeling and optimization in matlab," in In Proceedings of the CACSD Conference, Taipei, Taiwan, 2004.

[7] P. A. Parrilo, "Semidefinite programming relaxations for semialgebraic problems," Mathematical Programming, vol. 96, no. 2, pp. 293-320, 2003.

[8] J. B. Lasserre, Moments, Positive Polynomials and Their Applications. Imperial College Press, first edition, 2009.

[9] A. A. Ahmadi and G. Hall, "Dc decomposition of nonconvex polynomials with algebraic techniques," arxiv.org, 2015. [Online]. Available: https://arxiv.org/abs/1510.01518

[10] MathWorks, "Statistics and machine learning toolbox: User's guide (r2016b)," https://www.mathworks.com/help/pdf_doc/stats/stats.pdf, September 2016.

[11] G. Cimini, D. Bernardini, A. Bemporad, and S. Levijoki, "Online model predictive torque control for permanent magnet synchronous motors," in 2015 IEEE International Conference on Industrial Technology (ICIT), March 2015, pp. 2308-2313.

[12] P. Krause, O. Wasynczuk, S. Sudhoff, and I. P. E. Society, Analysis of electric machinery and drive systems, ser. IEEE Press series on power engineering. IEEE Press, 2002.

[13] D. P. Bertsekas, Convex Optimization Theory. Athena Scientific, 2009. 